\title{
What mechanisms dominate the activity of Geminid Parent (3200) Phaethon?
}

\author{
Liang Liang Yu, ${ }^{1 \star}$ Wing Huen Ip ${ }^{1,2}$ and Tilman Spohn ${ }^{3}$ \\ ${ }^{1}$ State Key Laboratory of Lunar and Planetary Science, Macau University of Science and Technology, Macau, China \\ ${ }^{2}$ Institute of Astronomy, National Central University, Jhongli, Taoyuan City 32001, Taiwan \\ ${ }^{3}$ Institute of Planetary Research, German Aerospace Center (DLR), Rutherfordstraße 212489 Berlin
}

Accepted 2018 October 28. Received 2018 October 5; in original form 2018 July 18

\begin{abstract}
A long-term sublimation model to explain how Phaethon could provide the Geminid stream is proposed. We find that it would take $\sim 6 \mathrm{Myr}$ or more for Phaethon to lose all of its internal ice (if ever there was) in its present orbit. Thus, if the asteroid moved from the region of a 5:2 or 8:3 mean motion resonance with Jupiter to its present orbit less than $1 \mathrm{Myr}$ ago, it may have retained much of its primordial ice. The dust mantle on the sublimating body should have a thickness of at least $15 \mathrm{~m}$ but the mantle could have been less than $1 \mathrm{~m}$ thick $1000 \mathrm{yr}$ ago. We find that the total gas production rate could have been as large as $10^{27} \mathrm{~s}^{-1}$ then, and the gas flow could have been capable of lifting dust particles of up to a few centimetres in size. Therefore, gas production during the past millennium could have been sufficient to blow away enough dust particles to explain the entire Geminid stream. For present-day Phaethon, the gas production is comparatively weak. But strong transient gas release with a rate of $\sim 4.5 \times 10^{19} \mathrm{~m}^{-2} \mathrm{~s}^{-1}$ is expected for its south polar region when Phaethon moves from $0^{\circ}$ to $2^{\circ}$ mean anomaly near perihelion. Consequently, dust particles with radii of $<\sim 260 \mu \mathrm{m}$ can be blown away to form a dust tail. In addition, we find that the large surface temperature variation of $>600 \mathrm{~K}$ near perihelion can generate sufficiently large thermal stress to cause fracture of rocks or boulders and provide an efficient mechanism to produce dust particles on the surface. The time-scale for this process should be several times longer than the seasonal thermal cycle, thereby dominating the cycle of appearance of the dust tail.
\end{abstract}

Key words: conduction-diffusion-methods: analytical - methods: numerical-minor planets, asteroids: individual: (3200) Phaethon.

\section{INTRODUCTION}

(3200) Phaethon, discovered by S.F. Green in 1983 October from data of the Infrared Astronomical Satellite (IRAS; Green 1983), is an intriguing Apollo-group Near-Earth Object with an unusual elliptical $(e \sim 0.89)$ orbit that crosses the orbits of Mars, Earth, Venus, and Mercury. The dynamical characteristics of Phaethon and of the Geminid meteoroids suggest that Phaethon may be the source of the Geminid meteor stream, with some of the meteoroids being ejected from Phaethon at its perihelion during the past $\sim 1000 \mathrm{yr}$ (Gustafson 1989). If true, Phaethon would have had a comet-like activity, at least for the past millennium.

Most meteoroid streams are of cometary origin (Jenniskens 2008) where mass-losses are driven by the sublimation of near-surface volatiles. Phaethon, judging from its dynamical characteristics, is an asteroid and is not likely to retain near-surface volatiles for a long time because of its periodically high surface temperature at perihelion. Its present perihelion distance of $\sim 0.14$ au suggests surface temperatures as high as $\sim 1000 \mathrm{~K}$, thus raising questions about the mechanisms for continued significant mass-loss from Phaethon.

Jewitt, Li \& (2013) detected Phaeton's tail in data taken by the STEREO spacecraft during two perihelion passages in 2009 and 2012, respectively, thereby confirming Phaeton's previously predicted activity. Hui \& Li (2017) again observed the dust tail during Phaethon's perihelion passage in 2016. The recurring of the tail at perihelion implies that it should have a periodic cause associated with the asteroid's orbital position. Jewitt et al. (2013) and Jewitt, Hsieh \& Agarwal (2015) explained the tails as being due to a combination of radiation pressure sweeping particles away and thermal disintegration of the asteroid surface caused by rotational thermal stress or thermal desiccation cracking, which seems to be the most convincing mechanisms at present. However, there still remains questions in this scenario.

First, it should be expected that similar small bodies with similar small perihelion distances as Phaethon should also have such dust 
tails, and may be expected to be associated with meteor streams as well. But Jewitt (2013) shows that other asteroids with similar small-perihelion distance do not have tails as Phaethon does.

Secondly, all of the three observations (Jewitt et al. 2013; Hui \& Li 2017) reported that the tail suddenly appeared at Phaeton's perihelion mean anomaly $=0^{\circ} \sim 1^{\circ}$ and continued for a very short time of $\sim 2 \mathrm{~d}$ (Li \& Jewitt 2013). This appears more like a shortterm outburst rather than the result of continuous solar radiation sweeping.

Moreover, both Jewitt et al. (2013) and Hui \& Li (2017) argued that the particles in Phaethon's tail were mainly small particles with sizes of $\sim 1 \mu \mathrm{m}$. These would be quickly swept away by the solar radiation pressure and thus can hardly account for the Geminids. The Geminid meteor stream consists of much larger particles with sizes ranging from $\sim 10 \mu \mathrm{m}$ to $\sim 1 \mathrm{~cm}$ (Yanagisawa et al. 2008; Borovička et al. 2010; Blaauw 2017). These unresolved questions imply that other mechanisms than those cited above may be responsible for the observed mass-loss from Phaethon.

Therefore, it has been speculated that Phaethon may still contain relatively pristine volatiles buried below the surface and that gaseous outburst near perihelion could eject large dust particles to supply the Geminid meteor stream (Boice \& Benkhoff 2015; Boice 2017). This idea is supported by the results of Licandro et al. (2007) that Phaethon's spectrum is consistent with that of B-type asteroids, similar to that of $\mathrm{CI} / \mathrm{CM}$ meteorites altered by aqueous activity and of hydrated minerals. de León et al. (2010) suggested that Phaethon may be a fragment of (2) Pallas because of their compositional similarities and dynamical connections. The hypothesis of a dynamical connection between Phaethon and Pallas is further supported by Todorović (2018), who finds a significant probability of $>10$ per cent for a fast delivery (on a time-scale of $\sim 1 \mathrm{Myr}$ ) of Phaethon from the region of a 5:2 or 8:3 mean motion resonance with Jupiter to its present orbit.

Generally, B-type small bodies, a subclass of the wider C-type, are mostly believed to be primitive, volatile-rich remnants from the early Solar system (Rivkin et al. 2002). The discovery of main-belt comets by Hsieh \& Jewitt (2006) further supports the idea that mainbelt objects could still have retained ice. Therefore, Pallas could be an icy body, and if Phaethon were an icy fragment of Pallas, it could have at least started in its present orbit with ice buried underneath its surface.

In this work, we aim to investigate whether ice could have been retained below the surface of Phaethon assuming that the latter is a fragment of Pallas, and whether sublimation-driven activity could explain the Phaethon-Geminids connection, as well as the tail observed during recent perihelion passages.

\section{THERMAL MODEL OF ICY SMALL BODIES}

\subsection{Dust-ice two-layer system}

Consider an icy small body of radius $R$ with an initial ice/dust mass ratio of $\chi_{0}$. If such a body is transferred to an orbit close enough to the Sun where the surface temperature gets high enough for the sublimation of ice to occur, then a dust mantle with porosity $\phi$ and tortuosity $\varsigma$ will gradually grow on its surface. And the ice front, the interface between the dust mantle and the icy interior at depth $h_{\mathrm{i}}$ (or radius $r_{\mathrm{i}}$ ), will gradually retreat towards the interior (compare Fig. 1).

In this system, there are three thermo-physical processes - heat flow, Knudsen gas flow, and sublimation/condensation, which can

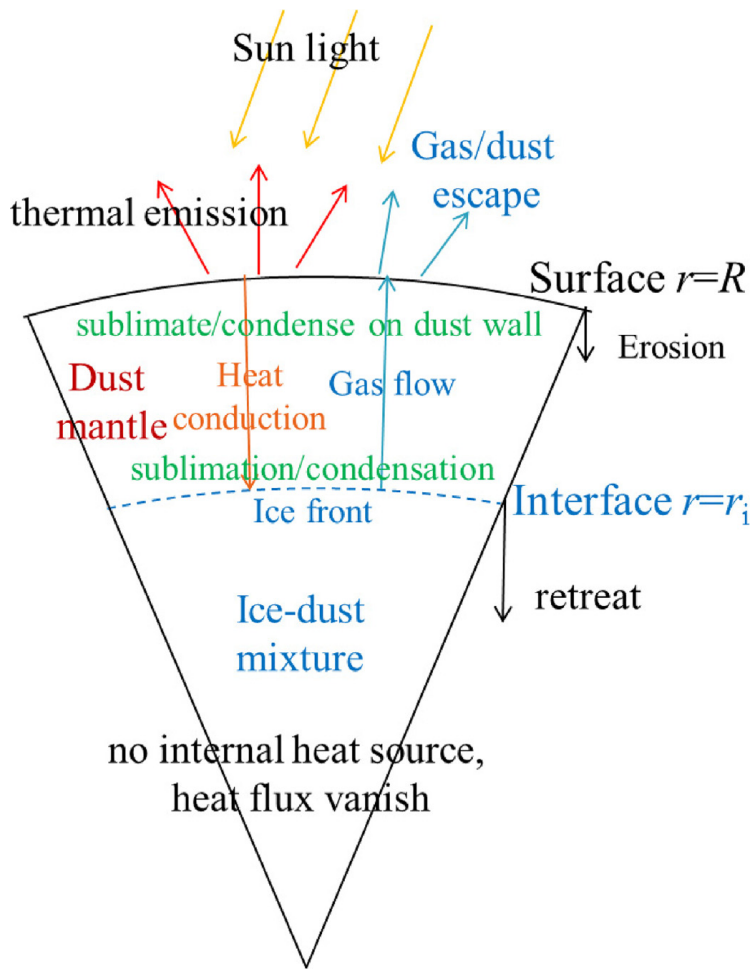

Figure 1. Dust layer on top of the ice-dust interior of an icy small body.

be described by the equations of thermal diffusion

$\rho c \frac{\partial T}{\partial t}+\nabla \cdot \boldsymbol{q}+c_{\mathrm{g}} \hat{m} \boldsymbol{j} \cdot \nabla T=Q$,

gas diffusion

$\frac{\partial n}{\partial t}+\nabla \cdot j=J \xi$

and sublimation/condensation

$\frac{\mathrm{d} \varphi}{\mathrm{d} t}=-\frac{\hat{m} J \xi}{\rho_{\mathrm{i}}}$.

In equations (1)-(3), $\rho$ is the solid mass density, $c$ is solid specific heat capacity, $T$ is temperature, a function of radial distance $r$ from the centre and latitude, $c_{\mathrm{g}}$ is the gas specific heat capacity, $\hat{m}$ is the gas molecular mass, $n$ is the number density of gas molecules, $\varphi$ is the volume fraction of ice, $\xi$ is the surface to volume ratio of the dust grain matrix,

$q=-\kappa \nabla T$

is the heat flux,

$\boldsymbol{j}=-\beta \nabla n$

is gas diffusion flux,

$Q=-\Delta H \hat{m} J \xi$

is the latent heat exchange caused by sublimation/condensation, where $\Delta H$ is the enthalpy of ice sublimation and is a function of temperature,

$J=\frac{1}{4} \tilde{v}_{\mathrm{th}}\left(n_{\mathrm{E}}-n\right)$ 
is the rate of sublimation/condensation per unit area,

$\tilde{v}_{\mathrm{th}}=\sqrt{\frac{8 k_{\mathrm{B}} T}{\pi \hat{m}}}$

is the mean thermal velocity, $n_{\mathrm{E}}$ is the equilibrium (or saturation) number density, which can be calculated from the temperature via the ideal gas law and the integral form of Clausius-Clapeyron equation

$n_{\mathrm{E}}=\frac{P_{\mathrm{E}}}{k_{\mathrm{B}} T}, P_{\mathrm{E}}=P_{0} \exp \left(-\frac{\Delta H_{\mathrm{m}}}{R_{\mathrm{g}}}\left(\frac{1}{T}-\frac{1}{T_{0}}\right)\right)$,

where $P_{0}=611 \mathrm{~Pa}, T_{0}=273.15 \mathrm{~K}, \Delta H_{\mathrm{m}}=\Delta H \hat{m} N_{\mathrm{A}}$ is the enthalpy of ice sublimation per mol, $R_{\mathrm{g}}$ is the gas constant, and $N_{\mathrm{A}}$ is Avogadro's number. In the case of an approximately constant sublimation temperature of about $210 \mathrm{~K}, \Delta H$ can be taken as constant:

$\Delta H \approx 2.83 * 10^{6} \mathrm{Jkg}^{-1}$.

The gas flow in the dust mantle can be treated as 'Knudsen diffusion' (diffusion of a rarefied gas), where the Knudsen diffusion coefficient $\beta$, for porous media can be estimated from (Schorghofer 2008)

$\beta=\left(\frac{\pi}{8+\pi} \frac{\phi}{1-\phi} \frac{b}{\zeta}\right) \tilde{v}_{\text {th }}$,

where $b$ is the mean grain radius, $\phi$ is porosity, and $\zeta$ is tortuosity.

The thermal conductivity $\kappa$ of the dust mantle as a function of temperature $T$, mean grain radius $b$ and porosity $\phi$ can be modelled following Gundlach \& Blum (2013):

$$
\begin{aligned}
\kappa(T, b, \phi)= & \kappa_{\text {solid }}\left(\frac{9 \pi}{4} \frac{1-\mu^{2}}{E} \frac{\gamma(T)}{b}\right)^{1 / 3} \cdot f_{1} e^{f_{2}(1-\phi)} \cdot \chi \\
& +8 \sigma \epsilon T^{3} \frac{e_{1} \phi}{1-\phi} b
\end{aligned}
$$

where $\kappa_{\text {solid }}$ is the thermal conductivity of the solid material, $\mu$ is Poisson's ratio, $E$ is Young's modulus, $\gamma(T)$ is the specific surface energy, $\varepsilon$ is the emissivity of the material, and $f_{1}, f_{2}, \chi$, and $e_{1}$ are best-fitting coefficients. For more details, we refer the reader to Gundlach \& Blum (2013).

\subsection{Long-term sublimation of buried ice}

We are considering the long-term evolution of icy small bodies, that is the evolution on a time-scale much longer than the orbital period. In this case, the thickness of the dust mantle $h_{i}$ by far exceeds the seasonal thermal skin depth $l_{\text {sst }}$

$h_{i}=R-r_{i} \gg l_{\mathrm{sst}} \sim 1 \mathrm{~m}$,

with $r_{i}$ the radial distance to the bottom of the dust mantle, and the model can be significantly simplified by taking the following assumptions:

(1) Although the surface temperature varies periodically due to rotation and orbital movement, the subsurface temperature below several seasonal thermal skin depths will be in a quasi-static equilibrium with the average solar insolation and thermal emission from the surface.

(2) The heat flow in the dust mantle from the surface to the ice sublimation front is mainly consumed by the latent heat of sublimation there.

(3) The difference between the subsurface temperature $\tilde{T}_{0}$ in equilibrium with the insolation and the temperature $T_{\mathrm{i}}$ of the ice front can be considered constant. This temperature difference continuously drives sublimation at the ice front.

The equilibrium subsurface temperature $\tilde{T}_{0}$ can be estimated by assuming radiation equilibrium at the surface

$\tilde{T}_{0}=\left[\frac{\left(1-A_{\mathrm{B}}\right) \tilde{F}}{\varepsilon \sigma}\right]^{1 / 4}$,

where $A_{\mathrm{B}}$ is the Bond albedo, $\varepsilon$ is the average thermal emissivity, and $\tilde{F}$ is the annual average solar insolation. $A_{\mathrm{B}}$ can be estimated from the geometric albedo $p_{\mathrm{v}}$ via

$A_{\mathrm{B}}=(0.29+0.684 G) p_{\mathrm{v}}$,

where $G$ is the slope parameter in the H,G magnitude system of Bowell et al. (1989). Because of rotation and the incidence angle of insolation varying with latitude $\theta$, both the annual average solar insolation $\tilde{F}$ and $\widetilde{T}_{0}$ will vary with latitude. Therefore, it is necessary to determine $\tilde{F}=\tilde{F}(\theta)$ for the actual orbit and rotation state.

To calculate the temperature $T_{\mathrm{i}}$ at the ice front $\left(r=r_{i}\right)$, we start with the energy balance equation at the ice front (in thermal equilibrium)

$\nabla \cdot \boldsymbol{q}=Q=-\Delta H \hat{m} J \xi$,

from which we obtain

$q_{\mathrm{i}}\left(r_{\mathrm{i}}\right)=-\Delta H \hat{m} J$,

which implies that the heat flow at the ice front is consumed by ice sublimation. For the heat flow at radius $r$ in the dust mantle, energy conservation further gives

$q=-\kappa \frac{\mathrm{d} T}{\mathrm{~d} r} \approx \frac{q_{\mathrm{i}} r_{\mathrm{i}}^{2}}{r^{2}}$.

We assume that the sublimation flux $J$ diffuses from the ice front to the surface. From mass conservation, we find the gas flow flux at a radial distance $r$ to be

$j=-\beta \frac{\mathrm{d} n}{\mathrm{~d} r} \approx \frac{J r_{\mathrm{i}}^{2}}{r^{2}}$.

Integrating both sides of equations (16) and (17), we obtain

$\left.T\right|_{r=R} ^{r=r_{\mathrm{i}}} \approx \int_{R}^{r_{\mathrm{i}}} \frac{q_{\mathrm{i}}}{\kappa} \mathrm{d}\left(\frac{1}{r}\right)$

$\left.n\right|_{r=R} ^{r=r_{\mathrm{i}}} \approx \int_{R}^{r_{\mathrm{i}}} \frac{J}{\beta} \mathrm{d}\left(\frac{1}{r}\right)$,

which further gives

$T_{\mathrm{i}}-\tilde{T}_{0} \approx r_{\mathrm{i}}\left(1-\frac{r_{\mathrm{i}}}{R}\right) \frac{q_{\mathrm{i}}}{\tilde{\kappa}}$,

$n_{\mathrm{i}}-n_{0} \approx r_{\mathrm{i}}\left(1-\frac{r_{\mathrm{i}}}{R}\right) \frac{J}{\tilde{\beta}}$,

assuming $\tilde{\kappa}$ and $\tilde{\beta}$ to be constant in the dust mantle with their average values calculated from equations (10) and (11).

For small bodies, the gravity is too low to prevent thermal escape of the water vapour molecules at the surface, thus we take

$n_{0} \approx 0$.

And at the ice front, because of sublimation,

$J=\frac{1}{4} \tilde{v}_{\mathrm{th}}\left(T_{\mathrm{i}}\right)\left(n_{\mathrm{E}}\left(T_{\mathrm{i}}\right)-n_{\mathrm{i}}\right)$, 
from which we obtain using equations (18) and (19)

$n_{\mathrm{i}}=\frac{r_{\mathrm{i}}\left(1-\frac{r_{\mathrm{i}}}{R}\right)}{r_{\mathrm{i}}\left(1-\frac{r_{\mathrm{i}}}{R}\right)+4 \beta / \tilde{v}_{\mathrm{th}}\left(T_{\mathrm{i}}\right)} n_{\mathrm{E}}\left(T_{\mathrm{i}}\right)$,

$J=\frac{\beta n_{\mathrm{E}}\left(T_{\mathrm{i}}\right)}{r_{\mathrm{i}}\left(1-\frac{r_{\mathrm{i}}}{R}\right)+4 \beta / \tilde{v}_{\mathrm{th}}\left(T_{\mathrm{i}}\right)}$.

Moreover, combining equations (15), (18), and (19), we find

$\tilde{T}_{0}=T_{\mathrm{i}}+\frac{\Delta H \hat{m} \tilde{\beta} n_{\mathrm{i}}\left(T_{\mathrm{i}}\right)}{\tilde{\kappa}}$,

which enables us to calculate the temperature $T_{\mathrm{i}}$ of the ice front from the equilibrium subsurface temperature $\tilde{T}_{0}$.

Here, we should note that when the ice front radius

$r_{\mathrm{i}} \rightarrow 0$ or $r_{\mathrm{i}} \rightarrow R$

then

$r_{\mathrm{i}}\left(1-\frac{r_{\mathrm{i}}}{R}\right) \rightarrow 0$,

implying that the number density $n_{\mathrm{i}}$, the temperature $T_{\mathrm{i}}$, and the sublimation rate $J$ at the ice front tend towards

$n_{\mathrm{i}} \rightarrow 0, T_{\mathrm{i}} \rightarrow \tilde{T}_{0}$, and, $J \rightarrow \frac{1}{4} \tilde{v}_{\mathrm{th}}\left(\tilde{T}_{0}\right) n_{\mathrm{E}}\left(\tilde{T}_{0}\right)$.

However, the Knudsen diffusion coefficient $\beta$ of a rarefied gas in the dust mantle generally satisfies

$\frac{4 \tilde{\beta}}{\tilde{v}_{\mathrm{th}}\left(T_{\mathrm{i}}\right)} \sim b \ll 1 \mathrm{~m}$.

Thus, when the ice front radius

$1 \mathrm{~m}<r_{\mathrm{i}}<R-1 \mathrm{~m}$,

the condition

$\frac{4 \tilde{\beta}}{\tilde{v}_{\mathrm{th}}\left(T_{\mathrm{i}}\right)} \ll 1<r_{\mathrm{i}}\left(1-\frac{r_{\mathrm{i}}}{R}\right)$

will always be true, so that in most of the interior of the small body,

$n_{\mathrm{i}} \approx n_{\mathrm{E}}\left(T_{\mathrm{i}}\right)$

$\tilde{T}_{0}=T_{\mathrm{i}}+\frac{\Delta H \hat{m} \tilde{\beta} n_{\mathrm{E}}\left(T_{\mathrm{i}}\right)}{\tilde{\kappa}}$,

and

$J \approx \frac{\tilde{\beta} n_{\mathrm{E}}\left(T_{\mathrm{i}}\right)}{r_{\mathrm{i}}\left(1-\frac{r_{\mathrm{i}}}{R}\right)}$.

According to equations (21) and (25), when the ice front radius reaches the value $r_{\mathrm{i}}=R / 2$, the term

$r_{\mathrm{i}}\left(1-\frac{r_{\mathrm{i}}}{R}\right)=\frac{R}{4}$,

will attend a maximum value while the sublimation rate $J$ attends a minimum value

$J_{\min }=\frac{4 \tilde{\beta} n_{\mathrm{E}}\left(T_{\mathrm{i}}\right)}{R}$.

Therefore, as the ice front $r_{\mathrm{i}}$ retreats from the surface to $R / 2$, the sublimation rate $J$ decreases from

$\frac{1}{4} \tilde{v}_{\mathrm{th}}\left(\tilde{T}_{0}\right) n_{\mathrm{E}}\left(\tilde{T}_{0}\right) \rightarrow \frac{4 \tilde{\beta} n_{\mathrm{E}}\left(T_{\mathrm{i}}\right)}{R}$.
For even deeper sublimation, when $r_{\mathrm{i}}$ moves from $R / 2$ to the body centre, the sublimation rate $J$ increases from

$\frac{4 \tilde{\beta} n_{\mathrm{E}}\left(T_{\mathrm{i}}\right)}{R} \rightarrow \frac{1}{4} \tilde{v}_{\mathrm{th}}\left(\tilde{T}_{0}\right) n_{\mathrm{E}}\left(\tilde{T}_{0}\right)$,

until all ice is exhausted.

With the sublimation rate $J$, the rate of retreat of the buried ice front can be calculated from

$$
\begin{aligned}
\frac{\mathrm{d} r_{\mathrm{i}}}{\mathrm{d} t} & =-\frac{\hat{m} J}{(1-\phi) \rho_{\mathrm{d}} \chi_{0}} \approx-\frac{1}{r_{\mathrm{i}}\left(1-\frac{r_{\mathrm{i}}}{R}\right)} \frac{\hat{m} \tilde{\beta} n_{\mathrm{E}}}{(1-\phi) \rho_{\mathrm{d}} \chi_{0}} \\
& \equiv-\frac{R_{r}}{r_{\mathrm{i}}\left(1-\frac{r_{\mathrm{i}}}{R}\right)},
\end{aligned}
$$

or

$\frac{\mathrm{d} h_{\mathrm{i}}}{\mathrm{d} t}=-\frac{\mathrm{d} r_{\mathrm{i}}}{\mathrm{d} t} \approx \frac{R_{r}}{r_{\mathrm{i}}\left(1-\frac{r_{\mathrm{i}}}{R}\right)}=\frac{R_{r}}{h_{\mathrm{i}}\left(1-\frac{h_{\mathrm{i}}}{R}\right)}$,

where $R_{r}$ is defined as

$$
\begin{aligned}
R_{r} & \equiv \frac{\hat{m} \tilde{\beta} n_{\mathrm{E}}}{(1-\phi) \rho_{\mathrm{d}} \chi_{0}} \\
& =\left(\frac{\pi}{8+\pi} \frac{\phi}{(1-\phi)^{2}} \frac{b}{\rho_{\mathrm{d}} \chi_{0} \varsigma}\right) \hat{m} \tilde{v}_{\mathrm{th}} n_{\mathrm{E}}\left(T_{\mathrm{i}}\right),
\end{aligned}
$$

in which

$\tilde{v}_{\mathrm{th}}=\frac{1}{2}\left(\tilde{v}_{\mathrm{th}}\left(\tilde{T}_{0}\right)+\tilde{v}_{\mathrm{th}}\left(T_{\mathrm{i}}\right)\right)$.

$R_{r}$ can be understood as the characteristic rate at which the sublimation front retreats, we thus name it 'retreating rate'. Because the saturation number density $n_{\mathrm{E}}$ depends exponentially on temperature (compare equation 9), the value of $R_{r}$ is mainly a function of the ice front temperature $T_{\mathrm{i}}$.

If the erosion of the surface due to gas drag can be ignored on the time-scale considered, $R=$ const. In the case of a stable orbit and rotation state, $\tilde{T}_{0}$ and $T_{\mathrm{i}}$ will be constant in time, so that, in addition, $R_{r}=$ const. By integrating both sides of equation (27) or (28), we obtain

$$
\left(\frac{r_{\mathrm{i}}^{2}}{2}-\frac{r_{\mathrm{i}}^{3}}{3 R}\right) \approx\left(\frac{r_{0}^{2}}{2}-\frac{r_{0}^{3}}{3 R}\right)-R_{r} t,
$$

Or

$$
\left(\frac{h_{\mathrm{i}}^{2}}{2}-\frac{h_{\mathrm{i}}^{3}}{3 R}\right) \approx\left(\frac{h_{0}^{2}}{2}-\frac{h_{0}^{3}}{3 R}\right)+R_{r} t,
$$

where $r_{0}$ and $h_{0}$ are the initial radius and depth to the ice front, respectively, and $r_{\mathrm{i}}$ and $h_{\mathrm{i}}$ are the radius and depth to the ice front, respectively, as functions of time $t$.

As a special case, if the depth to the ice front $h_{\mathrm{i}} \ll R$, we have

$\frac{\mathrm{d} h_{\mathrm{i}}}{\mathrm{d} t} \approx \frac{R_{r}}{h_{\mathrm{i}}\left(1-\frac{h_{\mathrm{i}}}{R}\right)} \approx \frac{R_{r}}{h_{\mathrm{i}}}$,

from which

$h_{\mathrm{i}} \approx \sqrt{h_{0}^{2}+2 R_{r} t}$

Moreover, if the initial radial distance from the centre of the ice front is $r_{0}=R$, then the time-scale to lose all the ice $\left(r_{\mathrm{i}}=0\right)$ is

$t_{\mathrm{L}}=\frac{R^{2}}{6 R_{r}}$ 
Table 1. Assumed physical parameters of the dust mantle of Pallas.

\begin{tabular}{lc}
\hline Property & Value \\
\hline Grain density $\rho_{\mathrm{d}}$ & $3000 \mathrm{~kg} \mathrm{~m}^{-3}$ \\
Mean Grain radius $b$ & $100 \mu \mathrm{m}$ \\
Tortuosity $\varsigma$ & 2 \\
Porosity $\phi$ & 0.5 \\
Ice/dust ratio $\chi_{0}$ & $0.1 \sim 0.2$ \\
\hline
\end{tabular}

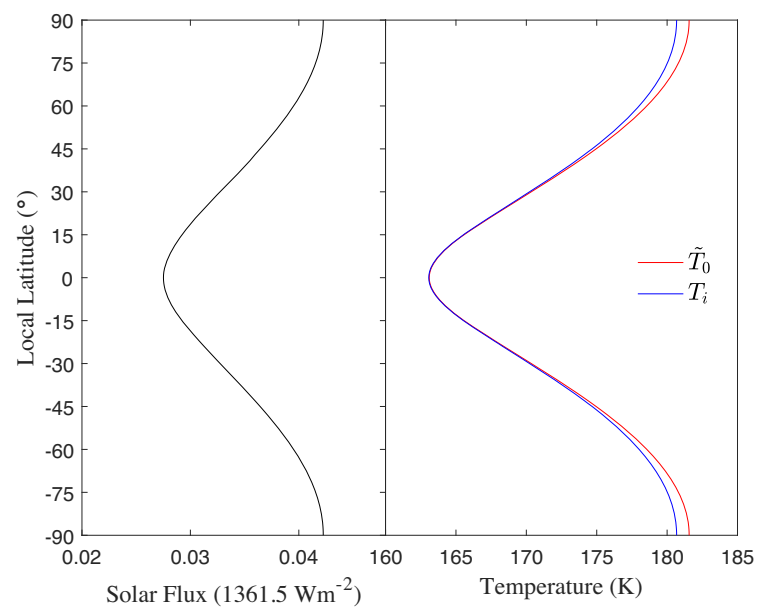

Figure 2. Left-hand panel: annual mean solar insolation as a function of local latitude on Pallas; right-hand panel: equilibrium subsurface temperature $\tilde{T}_{0}$ and ice sublimation front temperature $T_{\mathrm{i}}$ as functions of local latitude, calculated assuming a geometric albedo of $p_{\mathrm{v}}=0.1$.

\section{LONG-TERM THERMAL EVOLUTION}

\subsection{Could Phaethon once have contained ice?}

To discuss whether or not Phaethon may have retained ice through its history to the present day, we will have to know how and where the asteroid originated. If Phaethon formed as a fragment of Pallas, blown off by a relatively recent impact, for example, then the question would be whether Pallas could still have contained ice at the time of the recent Phaeton-forming collision after billions of years of evolution.

Pallas is a B-type asteroid most likely formed in the solar nebula beyond the snow line (Sasselov \& Lecar 2000) of the early Solar system. With an effective diameter of $\sim 545 \mathrm{~km}$, Pallas is large enough so that its orbit and rotation can be stable even on the timescale of the age of the Solar system. Thus, equation (31) can be applied to estimate the depth to any remaining ice in the interior. The orbital parameter values for Pallas can be found in the JPL Small-Body database and the rotation parameters have been well constrained by Carry et al. (2010) with a rotation period of $7.8134 \mathrm{~h}$ and angles to the rotation axis of $(-16.0,30.0)$ in the Ecliptic J2000 reference frame, implying a high axial tilt of $84 \pm 5^{\circ}$. Reasonable physical parameter values for the dust mantle are listed in Table 1.

The rotation axis of Pallas nearly lies in the orbital plane causing the polar regions to receive more solar insolation than the equatorial regions (left-hand panel in Fig. 2). The right-hand panel in Fig. 2 shows the equilibrium subsurface temperature $\tilde{T}_{0}$ on each local latitude of Pallas and the corresponding ice front temperature $T_{\mathrm{i}}$ derived from equation (24). The equatorial subsurface temperature is smaller than the subsurface temperature at higher latitudes,

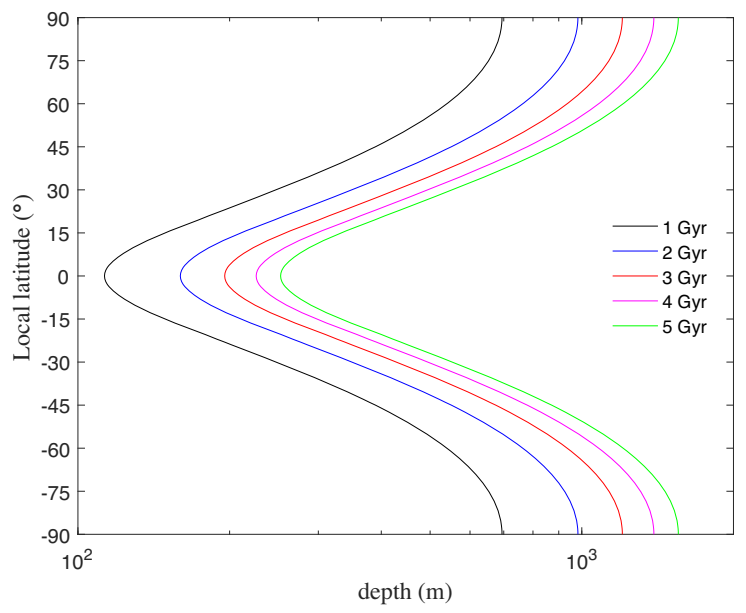

Figure 3. Depth to the ice front in Pallas calculated from the present model.

implying that the loss rate of ice on the equator should be smaller than the loss rate at high latitudes.

Given the ice sublimation front temperature, the inward retreating rate $R_{r}(\theta)$ can be estimated for each latitude, and then the secular evolution of the ice front can be evaluated as shown in Fig. 3. Along the equator of Pallas, the inward retreating rate $R_{r}$ of the ice front is at a minimum value of $\sim 10^{-13} \mathrm{~m}^{2} \mathrm{~s}^{-1}$, suggesting that the time for losing all ice would be

$t_{\mathrm{L}} \sim \frac{R^{2}}{6 R_{r}} \sim 10^{7} \mathrm{Gyr}$.

This time is much longer than the age of the Solar system. Moreover, from Fig. 3, we find that even on Gyr time-scales, the depth to the ice front would still be within $\sim 100-1000$ m of the surface, suggesting that the dust mantle would have a thickness in the range of several hundred metres at most (because we do not consider any losses of dust mantle due to gas escape). This thickness of the dust mantle is much smaller than the size of Phaethon. Thus, if Phaethon were a fragment of Pallas formed by a giant impact, it would be likely that the bulk of Phaethon would contain ice, suggesting that Phaethon could have started as an icy body.

\subsection{Can Phaethon have retained ice to the present day?}

To answer the question of whether or not Phaethon can still retain ice in its interior (assuming it formed as an icy body), we would have to know its orbital evolution. We would then be able to model its thermal evolution as a consequence of its time varying insolation and trace the secular inward motion of the possible ice front. Unfortunately, it is difficult to exactly reconstruct the orbital evolution of a small body like Phaethon because the orbit was likely not stable. Moreover, if we assume Phaethon to be a fragment of Pallas, its orbit may have changed so much that its thermal history becomes almost impossible to reconstruct. However, because Phaethon is in a retrograde orbit, it is conceivable that its semimajor axis is continuously decreasing because of the Yarkovsky effect. The average subsurface temperature should then be increasing with time. Thus, the present equilibrium subsurface temperature, which can be easily calculated from the present orbit and rotation, can be used to place an upper limit on the past subsurface temperature.

We use the orbital parameters of Phaethon as published in the JPL Small-Body database and the rotation parameters derived by 
Table 2. Assumed physical parameters of Phaethon's dust mantle.

\begin{tabular}{lc}
\hline Property & Value \\
\hline Grain density $\rho_{\mathrm{d}}$ & $3000 \mathrm{~kg} \mathrm{~m}^{-3}$ \\
Mean Grain radius $b$ & $500 \mu \mathrm{m}$ \\
Tortuosity $\varsigma$ & 2 \\
Porosity $\phi$ & 0.4 \\
Ice/dust ratio $\chi_{0}$ & $0.1 \sim 0.2$ \\
\hline
\end{tabular}

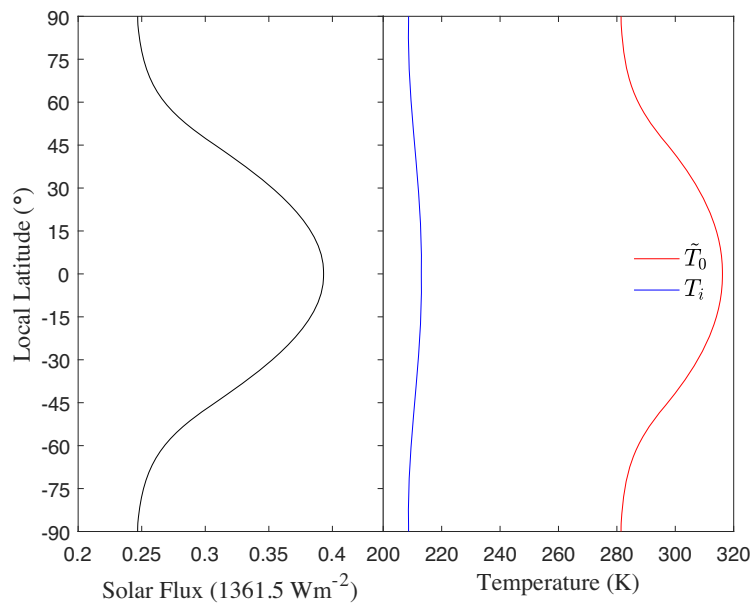

Figure 4. Left-hand panel: annual mean solar insolation as a function of local latitude on Phaethon; right-hand panel: equilibrium subsurface temperature $\tilde{T}_{0}$ and ice front temperature $T_{\mathrm{i}}$ of Phaethon, assuming a geometric Albedo of $p_{\mathrm{v}}=0.122$.

Hanuš et al. (2016) - rotation period $P_{\mathrm{rt}}=3.604 \mathrm{~h}$ and orientation $\left(\lambda=319^{\circ}, \beta=-39^{\circ}\right)$ in the Ecliptic coordinate system.

The physical parameter values for the dust mantle on Phaethon should be slightly different from those for Pallas because Phaethon has a much larger average surface thermal inertia of $\sim 600$ $\mathrm{Jm}^{-2} \mathrm{~s}^{-0.5} \mathrm{~K}^{-1}$ (Hanuš et al. 2016), suggesting a larger density and/or larger mean grain size. The physical properties of the Geminids may provide useful information on Phaethon's dust mantle if we assume that the Geminids do come from Phaethon. Borovička et al. (2010) found the bulk density of the Geminids to be $\sim 2600 \mathrm{~kg} \mathrm{~m}^{-3}$, indicating a 'microporosity' of $\sim 13$ per cent for a material density of $\sim 3000 \mathrm{~kg} \mathrm{~m}^{-3}$ (or anything reasonably close to that). Here, we use $\sim 3000 \mathrm{~kg} \mathrm{~m}^{-3}$ for the material density of dust grains that form the dust mantle, but assume a 'macroporosity' of $\sim 40$ per cent, somewhat smaller than that used for Pallas, considering that sintering may have reduced the dust mantle porosity.

The recent polarization measurements of Ito et al. (2018) suggest that grains of $\sim 150 \mu \mathrm{m}$ radius may characterize Phaethon's surface. Considering that the grains on the very surface may be easier to fracture due to space weathering or thermal stress, whereas grains in the subsurface dust mantle may aggregate and grow by sintering, we expect the average grain size on the surface to be smaller than the mean grain size in the subsurface dust mantle, if the formed dust mantle is enough thick ( $>1 \mathrm{~m}$ for example). Therefore, we assume a mean grain size of $\sim 500 \mu \mathrm{m}$ for the dust mantle. We list our chosen parameter values in Table 2.

The equilibrium subsurface temperature $\tilde{T}_{0}$ and the ice front temperature $T_{i}$ calculated from our model are shown in Fig. 4. The ice front temperature $T_{i}(208-213 \mathrm{~K})$ is found significantly lower than the equilibrium subsurface temperature $\tilde{T}_{0}(281-316 \mathrm{~K})$, in-

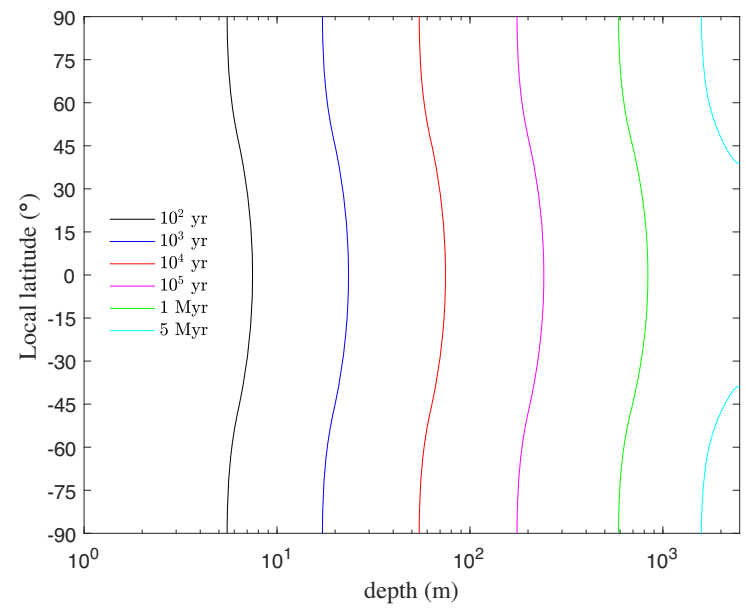

Figure 5. Phaethon: secular inward motion of the ice front.

dicating a larger temperature gradient in the dust mantle than we found for Pallas and thus a larger rate of energy consumption by ice sublimation.

As Fig. 4 shows, the ice front temperature $T_{i}$ varies little with latitude. Considering that the rotation axis may have changed randomly over Phaethon's dynamical history (Hanuš et al. 2016), it is thus likely that the latitudinal variation of the ice front temperature has not had any particular significance. Therefore, we use an average ice front temperature of

$T_{\mathrm{i}} \sim 210 \mathrm{~K}$

in the following. The inward retreating rate of the ice front can then be estimated to be

$R_{r} \sim 5.68 \times 10^{-9} \mathrm{~m}^{2} \mathrm{~s}^{-1}$,

along with the time of losing all ice of

$t_{\mathrm{L}} \sim \frac{R^{2}}{6 R_{r}} \sim 6 \mathrm{Myr}$

As a consequence, if Phaethon was to retain a significant fraction of its initial ice, then it must have been in its present orbit less than about 6 Myr. If the asteroid is a fragment of Pallas, then as Todorović (2018) shows, its orbit may have evolved from a 5:2 or a 8:3 mean motion resonance with Jupiter to its present orbit in $<\sim 1$ Myr. Fig. 5 shows that the depth to the ice front can be $<600 \mathrm{~m}$ if Phaethon arrived at its present orbit no longer than $1 \mathrm{Myr}$ ago. Therefore, it is totally possible that Phaethon still retains ice buried in its interior.

\subsection{Mean water gas production rate}

Assuming ice in the interior of Phaethon, we can predict the gas production rate, which would be the key parameter to relate our model to observation. With an ice front temperature of $T_{\mathrm{i}} \sim 210 \mathrm{~K}$, the sublimation rate $J$ at the ice front can be related to the depth to the ice front $h_{\mathrm{i}}$ using equation (21). The total production rate $J_{t}$ can then be estimated using

$J_{t}=4 \pi\left(R-h_{\mathrm{i}}\right)^{2} J$

In the upper panel of Fig. 6, we show how the mean sublimation rate $J$ at the ice front relates to the depth to the ice front $h_{\mathrm{i}}$. The sublimation rate $J$ initially decreases with $h_{\mathrm{i}}$ increasing from zero to $R / 2$. This is caused by the heat flow (and thereby the sublimation 

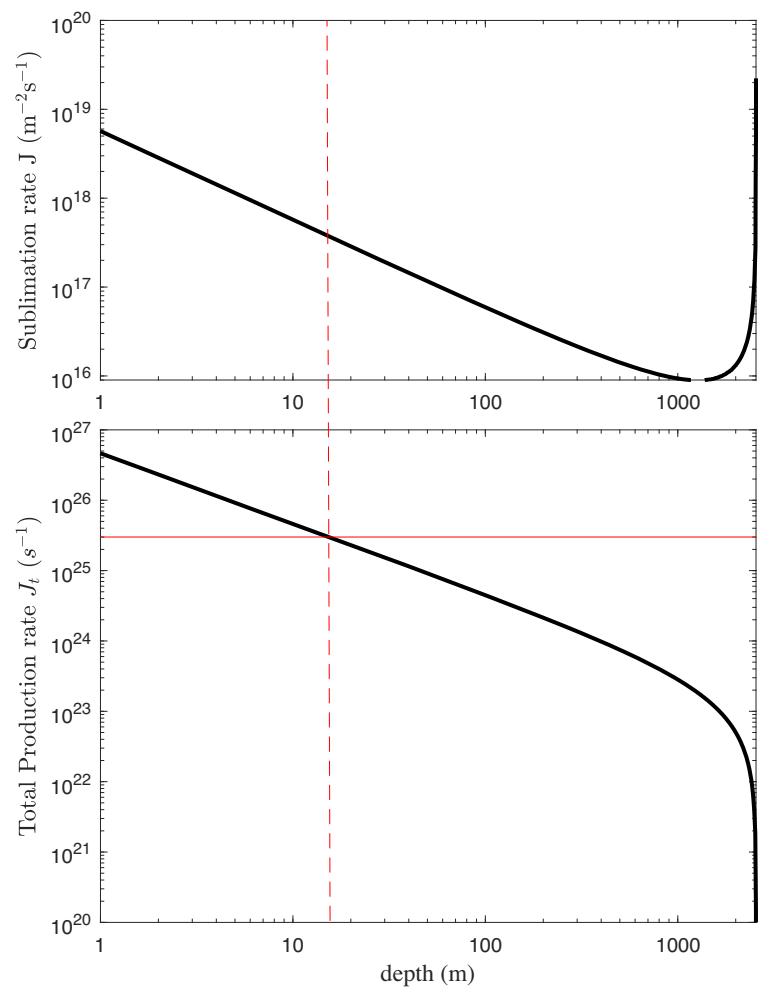

Figure 6. Phaethon: upper panel - sublimation rate $J$ at the ice front as a function of the depth to the ice sublimation front; lower panel - total production rate $J_{t}$.

rate) decreasing with increasing $h_{\mathrm{i}}$ at approximately constant temperature difference between the surface and the sublimation front $\left(\tilde{T}_{0}-T_{i}\right)$. At depths greater than $R / 2$, the spherical geometry effect begins to dominate and causes the heat flow per unit area on the sublimation front to increase as $\left(R-h_{\mathrm{i}}\right)$ decreases. The sublimation rate increases along with the heat flow and the remainder of the ice near the centre will be lost quickly.

The lower panel of Fig. 6 shows the mean total gas production rate $J_{t}$ as a function of depth to the sublimation front. Given observational constraints, we could use the figure to estimate the thickness of the dust mantle above the sublimation front. However, up to date, no ground-based telescope has ever observed a gas coma around Phaethon. Thus, the total water gas production $J_{t}$ from Phaethon must be

$J_{t}<\sim 3 \times 10^{25} \mathrm{~s}^{-1}$

(table III in Chamberlin et al. 1996), implying that the present-day depth to the ice front should be at least $\sim 15 \mathrm{~m}$.

On the other hand, according to Fig. 5, the ice front can move from $\sim 1 \mathrm{~m}$ depth to $\sim 20 \mathrm{~m}$ in about $\sim 10^{3} \mathrm{yr}$. Thus, if we assume the ideal case that the ice front is at $15 \mathrm{~m}$ presently, then it is possible that

$h_{\mathrm{i}}<1 \mathrm{~m}$

for Phaethon $\sim 10^{3}$ yr ago.

The long-term model will not be applicable for $h_{\mathrm{i}}<1 \mathrm{~m}$ because differences to the short-term thermal cycle would be significant. Nevertheless, we can argue that the total water production rate could have been $J_{t}>10^{27} \mathrm{~s}^{-1}$, which would be enough to supply the Geminids stream.

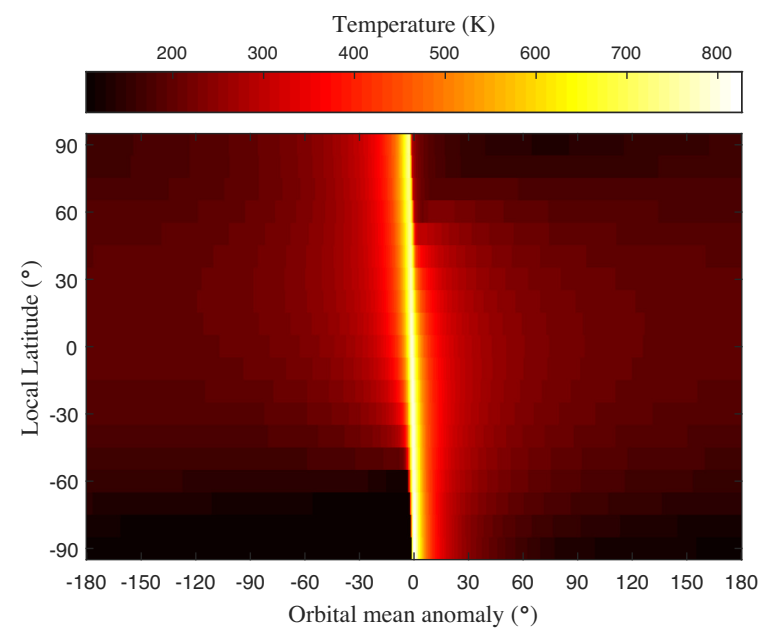

Figure 7. Phaethon: along-orbit variation of the diurnally averaged surface temperature as a function of local latitude.

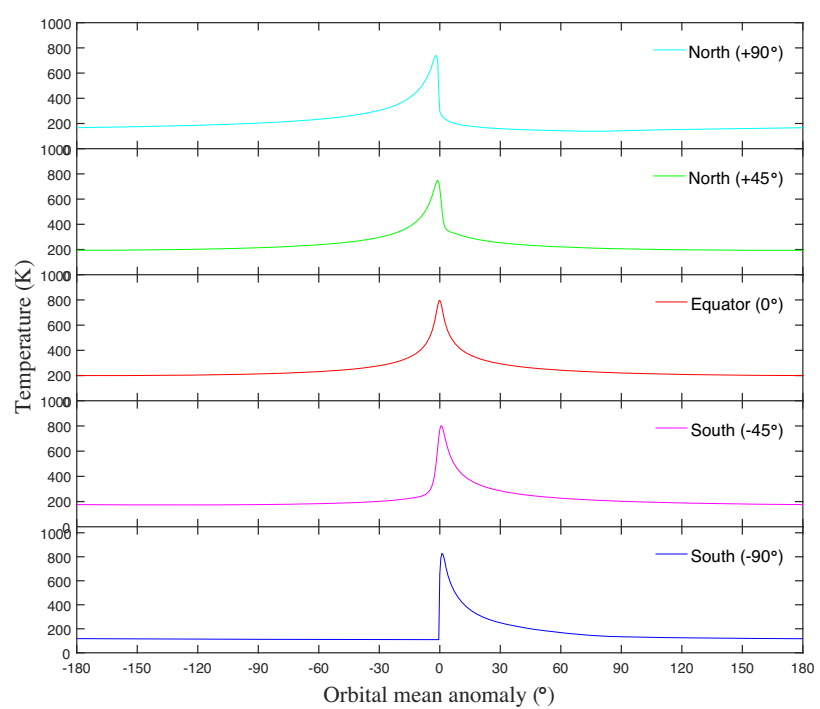

Figure 8. Along-orbit variation of the diurnally averaged surface temperature at the north pole, the equator and the south pole of Phaethon.

\section{SHORT-TERM THERMAL CYCLE}

\subsection{Seasonal and diurnal temperature cycle}

Considering the large eccentricity of Phaethon's orbit, it is expected that the surface temperature will vary considerably between perihelion and aphelion.

In Fig. 7, we present a map of Phaethon's surface temperature as a function of local latitude and mean anomaly. The temperature plotted has been averaged over one rotational period, each. As Fig. 7 shows, the surface temperature increases sharply near perihelion, in particular for the south polar region $\left(-50^{\circ}\right.$ to $-90^{\circ}$ latitude), while for the north polar region temperature increases more gradually but decreases rapidly after perihelion passage.

The difference in temperature between the two polar regions is also shown in Fig. 8 and compared with the temperature variation at mid-latitude and at the equator. The seasonal effect is significant at all latitudes, causing temperature variations $>600 \mathrm{~K}$, but is strongest at the south pole where temperature rapidly increases by $\sim 700 \mathrm{~K}$ 

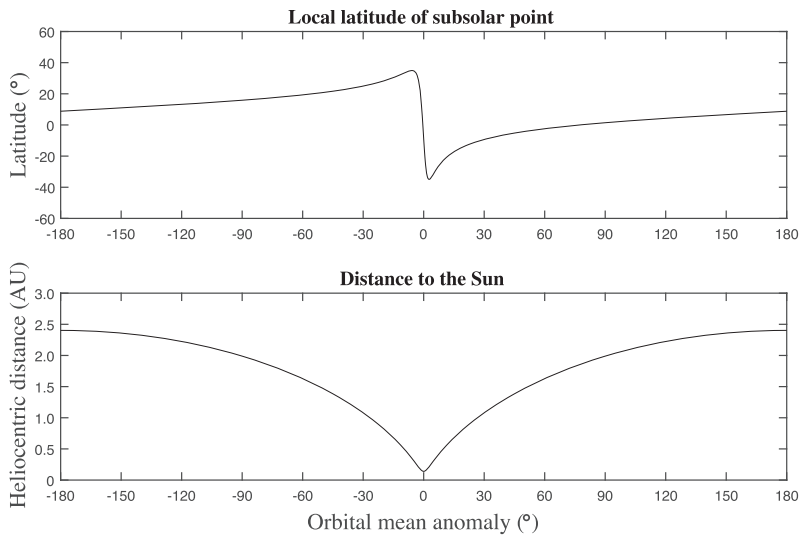

Figure 9. Upper panel: along-orbit variation of the latitude of the subsolar point on Phaethon; lower panel: along-orbit variation of the heliocentric distance to Phaethon.

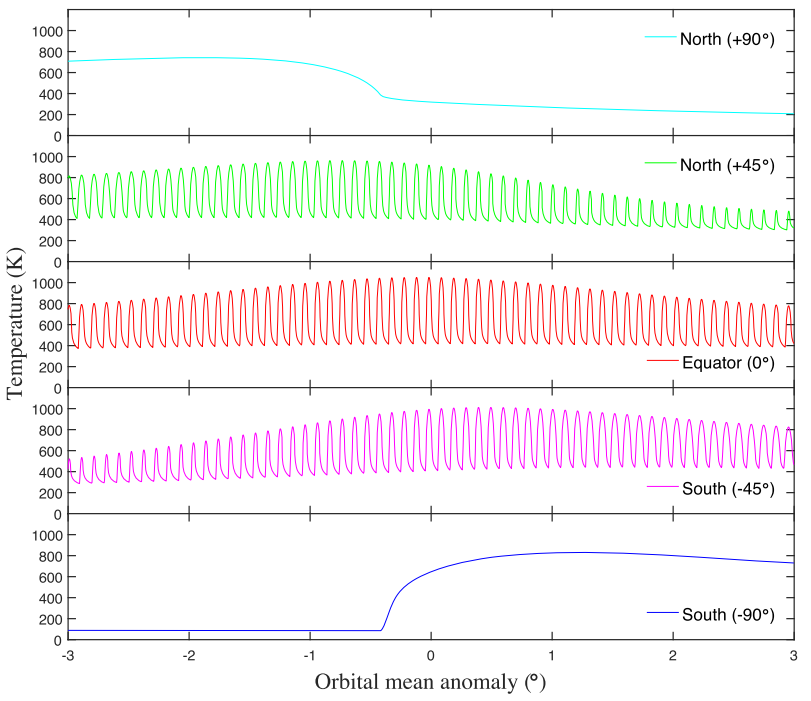

Figure 10. Surface temperature variation at the north pole, the equator and the south pole near perihelion for mean anomaly values between $-3^{\circ}$ and $+3^{\circ}$ showing both the effects of rotation and orbital motion.

in about $2 \sim 3 \mathrm{~d}$ near perihelion. These temperature variations can be explained as the results of the swift shift of the subsolar point from the Northern to the Southern hemisphere along with the rapid decrease of the heliocentric distance near perihelion (compare Fig. 9).

Fig. 10 shows the variation of the surface temperature near perihelion in more detail including variations caused by rotation. As expected, rotation effects can be neglected at the poles but become significant at low latitudes where the diurnal temperature variation can also be as large as $\sim 600 \mathrm{~K}$.

\subsection{Sublimation/condensation cycle near the south pole}

According to Fig. 8, the temperature at the south polar region will be sufficiently high to drive sublimation only between about $0^{\circ}$ and $60^{\circ}$ mean anomaly, equivalent to $\sim 1 / 6$ of the orbit. For the other $\sim 5 / 6$ of the orbit, the surface temperature keeps lower than $100 \mathrm{~K}$. Any outflowing gas will condense in a near-surface layer of $\sim l_{\text {stt }} / 5 \approx 20 \mathrm{~cm}$ thickness, and the condensed ice will reach a volume fraction of $\sim 0.2$ per cent, there. This is shown in Fig. 11 where a simulated cycle of sublimation and condensation is presented.
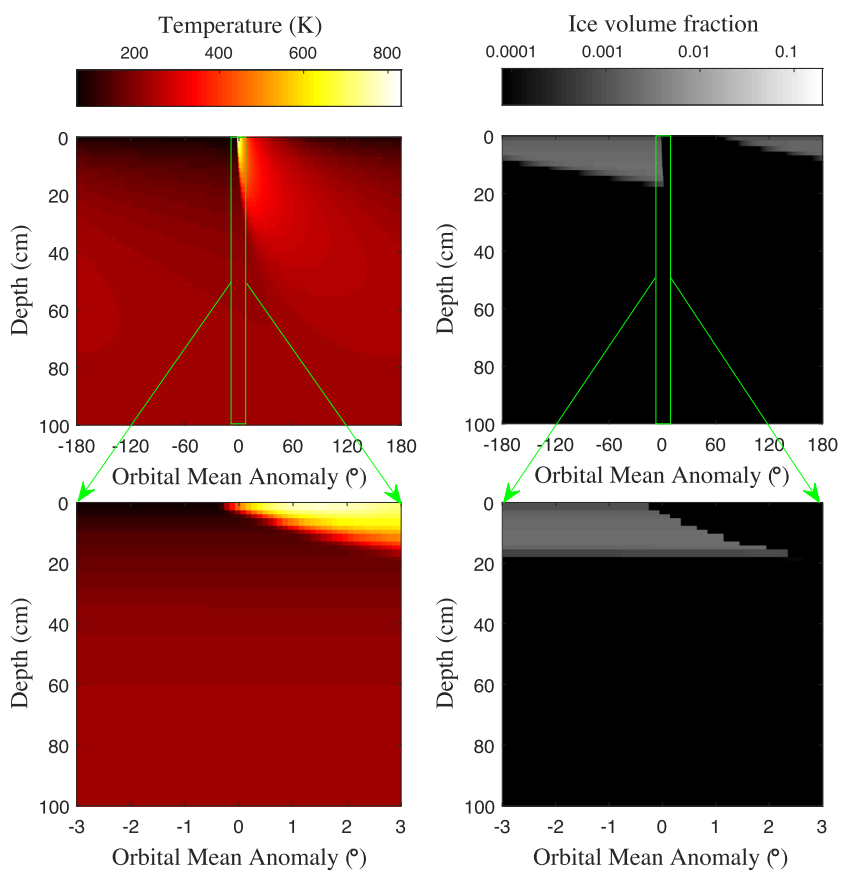

Figure 11. Simulation of a sublimation/condensation cycle on the south pole of Phaethon. The initial ice/dust mass ratio $\chi_{0}$ is assumed to be 0.15 (corresponding to initial ice volume fraction $\sim 0.3$ ) and the initial dust mantle thickness $h_{\mathrm{i}}=15 \mathrm{~m}$.

From Fig. 11, we further find that the condensed ice will be quickly sublimated near perihelion within $\sim 1 / 180$ of the orbital period $(\sim 2.9 \mathrm{~d})$. This transient sublimation pulse can generate a comparatively large gas outflow, $J_{\mathrm{s}, \max }$, which can be estimated using

$J_{\mathrm{s}, \max } \sim 180 \times \frac{5}{6} \tilde{J}_{\mathrm{s}}=150 \tilde{J}_{\mathrm{s}}$,

where $\tilde{J}_{\mathrm{s}}$ is the annual mean outflow water flux.

Considering the upper-limit case discussed in Section 3.3,

$\tilde{J}_{\mathrm{s}}<\frac{3 \times 10^{25} \mathrm{~s}^{-1}}{4 \pi R^{2}} \sim 3 \times 10^{17} \mathrm{~m}^{-2} \mathrm{~s}^{-1}$

for present Phaethon and

$\tilde{J}_{\mathrm{s}} \sim \frac{10^{27} \mathrm{~s}^{-1}}{4 \pi R^{2}} \sim 10^{19} \mathrm{~m}^{-2} \mathrm{~s}^{-1}$

$10^{3} \mathrm{yr}$ ago. The maximum outflow water flux near perihelion is then $J_{\mathrm{s}, \max }<\sim 4.5 \times 10^{19} \mathrm{~m}^{-2} \mathrm{~s}^{-1}$ at present and $J_{\mathrm{s}, \max } \sim 1.5 \times$ $10^{21} \mathrm{~m}^{-2} \mathrm{~s}^{-1}$ a thousand years ago.

\subsection{Dust acceleration}

The outflow gas can blow away small dust particles, but the important question is, how large a particle can be lifted off the surface? To answer this question, we proceed to compare the gravity force acting on a particle to the gas drag force.

Assume a spherical dust particle with effective radius $b_{\mathrm{d}}$ and mean density $\rho_{\mathrm{d}}$. Then, considering rotation, the gravity force on a dust particle at local latitude $\theta$ can be expressed as

$F_{\mathrm{G}}=\frac{4}{3} \pi b_{\mathrm{d}}^{3} \rho_{\mathrm{d}}\left(R \omega^{2} \cos ^{2} \theta-\frac{G M}{R^{2}}\right)$,

where $R$ is the radius of the host body and $\omega$ its rotation angular velocity. 
Table 3. Material properties for pyroxene and plagioclase (Molaro, Byrne \& 2015).

\begin{tabular}{lccc}
\hline Properties & Symbol & Pyroxene & Plagioclase \\
\hline Young's modulus(GPa) & $E$ & 175 & 85 \\
Possion's ratio & $\gamma$ & 0.23 & 0.33 \\
Linear expansion coefficient & $\alpha$ & $0.8 \times 10^{-5}$ & $0.4 \times 10^{-5}$ \\
$K^{-1}$ & & & \\
\hline
\end{tabular}

The gas drag force on the dust particle can be estimated from

$F_{\text {drag }}=\frac{\mathrm{d} P_{\mathrm{M}}}{\mathrm{d} t} \sim \frac{\hat{m} \tilde{v}_{\mathrm{th}} \cdot C_{\mathrm{d}} \pi b_{\mathrm{d}}^{2} \cdot J_{\mathrm{s}} \mathrm{d} t}{\mathrm{~d} t}=C_{\mathrm{d}} \pi b_{\mathrm{d}}^{2} \hat{m} \tilde{v}_{\mathrm{th}} J_{\mathrm{s}}$,

where $P_{\mathrm{M}}$ is the momentum of a dust particle, $C_{\mathrm{d}}$ is the drag coefficient, $\tilde{v}_{\text {th }}$ is the thermal velocity of the gas molecules at the surface, and $J_{\mathrm{s}}$ is the outflow number flux. Thus, the critical size of a dust particle that can be lifted off by the gas flow can be estimated from

$0=F_{\mathrm{G}}+F_{\text {drag }}$,

giving

$b_{\max } \sim \frac{\hat{m} v_{\text {th }} J_{\mathrm{s}}}{\rho_{\mathrm{d}}\left(\frac{G M}{R^{2}}-R \omega^{2} \cos ^{2} \theta\right)}$,

if using $C_{\mathrm{d}} \sim 4 / 3$ (Epstein 1924; Podolak et al. 2016) for spherical dust particles.

For present-day Phaethon, considering a maximum surface temperature of $\sim 800 \mathrm{~K}$ near perihelion, but a steady mean gas flux of

$\tilde{J}_{\mathrm{s}}<\sim 3 \times 10^{17} \mathrm{~m}^{-2} \mathrm{~s}^{-1}$,

the critical radius $b_{\max }$ is only about $1.8 \mu \mathrm{m}$. However, if considering the transient strong gas flow near perihelion,

$J_{\mathrm{s}, \max }<\sim 4.5 \times 10^{19} \mathrm{~m}^{-2} \mathrm{~s}^{-1}$,

we find $b_{\max }$ to be about $\sim 260 \mu \mathrm{m}$, still assuming a maximum surface temperature of $\sim 800 \mathrm{~K}$. In addition, for Phaethon $10^{3} \mathrm{yr}$ ago, we can easily estimate $b_{\max }$ to be as large as $1 \mathrm{~cm}$. These estimates are well consistent with the typical sizes $(\sim 10 \mu \mathrm{m}$ to $\sim 1 \mathrm{~cm}$ ) of the Geminid stream particles.

Our model predicts that for present-day Phaethon the transient gas outburst will only occur at the south polar region and that it will last only for a short time of $\left(<P_{\text {orbit }} / 180 \sim 2.9 \mathrm{~d}\right)$. Large dust particles can be blown away during perihelion passage only, for mean anomaly between $0^{\circ}$ and $2^{\circ}$. This is consistent with observation, since the dust tails reported by Jewitt et al. (2013) and Hui \& Li (2017) appeared at mean anomaly between $0^{\circ}$ and $1^{\circ}$ and lasted only $\sim 2 \mathrm{~d}$.

\subsection{Thermal stess near perihelion}

Large temperature variation can generate strong thermal stress, probably causing fracture of rocks or boulders. Using Hooke's law, we can estimate the thermal stress via

$\sigma_{\mathrm{th}}=E \Delta l=E \alpha_{\mathrm{th}} \Delta T$,

where $E$ is Young's modulus, and $\alpha_{\text {th }}$ is the linear coefficient of thermal expansion.

We estimate the thermal stress that will be generated by a temperature variation $\Delta T>600 \mathrm{~K}$ for pyroxene and plagioclase as two representative examples. Their mechanical and thermal properties are well known and are listed in Table 3.
The thermal stress induced by a temperature variation of $\Delta T>$ $600 \mathrm{~K}$ will be $\sigma>840 \mathrm{MPa}$ for pyroxene and $\sigma>204 \mathrm{MPa}$ for plagioclase. The fatigue strength of typical rock has been measured to be of the order of $<200 \mathrm{MPa}$ (Krokosky \& Husak 1968). Thus, a temperature variation of $\Delta T>600 \mathrm{~K}$ on the surface of Phaethon during each perihelion passage can be expected to cause cracks in typical rock. These cracks can propagate into the rock due to subsequent continuous thermal cycling and may lead to fracture and the production of dust particles over the course of time. However, this mechanism of dust formation will occur on time-scales longer than the seasonal cycle of sublimation and condensation. Therefore, the dust particles on the surface that can be blown away to form an observable tail are expected to become gradually exhausted with time. The high average surface thermal inertia $\sim 600 \mathrm{Jm}^{-2} \mathrm{~s}^{-0.5} \mathrm{~K}^{-1}$ of Phaethon (Hanuš et al. 2016) is similar to that of Itokawa, suggesting that there should be little dust on the surface of the asteroid. The cycle of the appearance of the tail would then be predicted to depend more on the time-scale of dust formation than gas release.

\section{DISCUSSION AND CONCLUSION}

Because Phaethon is hitherto the only small body that is dynamically associated with the Geminid stream, it has been widely accepted as its parent body, although the mechanism of how Phaethon would supply the Geminid stream has remained unclear. Here, on the basis of theoretical considerations, we find that Phaethon could have retained ice buried underneath the surface. We found that the tails reported in Jewitt et al. (2013) and Hui \& Li (2017) can be explained by transient gas outbursts near perihelion due to cycles of sublimation and condensation. The proposed mechanism would be able to supply the Geminid stream for the past $\sim 1000 \mathrm{yr}$, even though a comet-like coma has not been detected. The model implies that Phaethon may have been transferred to its present orbit $\sim 1000 \mathrm{yr}$ ago.

The feasibility of the proposed model depends on the validity of two assumptions: (1) Phaethon comes from the main belt as an initially icy body, possibly a fragment of Pallas; (2) Phaethon was delivered from the main belt to its present orbit about $\sim 1000 \mathrm{yr}$ ago. Although these two assumptions have support from some previous works, there exist other arguments against the assumptions. For example, Hanuš et al. (2016) did a backward orbit calculation of Phaethon for the past 1 Myr. They find only slight changes $\sim 10$ per cent of the semimajor axis, suggesting that Phaethon may have remained in its present orbit for several Myrs. Takir et al. (2018) found the spectra of Phaethon obtained during the night of 2017 December 12 to be featureless in the $3 \mu \mathrm{m}$ region, suggesting that its surface may be unhydrated, whereas Pallas exhibits a sharp $3 \mu \mathrm{m}$ band. Still, the data of Takir et al. (2018) cannot rule out that the surface of Phaethon is covered by a thick dry dust mantle below which ice may exist.

On the basis of our modelling, we conclude the following:

(1) It is possible, for present-day Phaethon, to still retain buried water ice in its interior; the dry dust mantle should have a thickness of $>15 \mathrm{~m}$ at least. Sublimation of the buried ice is too weak to generate a coma observable with present ground-based instruments.

(2) A thousand years before present, Phaethon's dust mantle could have had a thickness $<1 \mathrm{~m}$, which would have allowed Phaethon to be sufficiently active to supply the Geminid stream up to the present. If true, Phaethon may have been transferred from the main belt to its present orbit about $10^{3} \mathrm{yr}$ ago. 
We propose the following mechanisms to explain the relation to the Geminid stream and the observed dust tail:

(1) Sublimation of ice below the growing dust mantle could have provided the Geminid particles during the past millennium. A significant sublimation/condensation cycle is predicted for Phaethon's south polar region even today. The sublimation/condensation cycle would lead to transient gas outbursts during perihelion passage (mean anomaly between $0^{\circ}$ and $2^{\circ}$ ) capable of blowing away dust particles and explain the observed tail.

(2) The large temperature variation near perihelion could induce sufficiently large thermal stress to cause fracture of rocks or boulders and would be an efficient mechanism to produce dust particles on the surface which would then be blown away by out flowing gas to form the dust tail. But the time-scale of this dust producing process should be longer than the seasonal cycle of water sublimation and condensation and may dominate the cycle of appearance of Phaethon's tail.

Of course, the proposed mechanisms need to be further examined by new observation and in situ detection, especially precise constraints on the total gas production rate, from which we should be able to estimate how much ice Phaethon really contains. The proposed JAXA/ISAS DESTINY ${ }^{+}$mission to Phaethon would certainly bring new insight to understand the origin and evolution of Phaethon as well as its connection to the Geminid stream.

\section{ACKNOWLEDGEMENTS}

This work was financially supported by the Science and Technology Development Fund of Macao (Grants No. 119/2017/A3, 061/2017/A2), and the Strategic Priority Research Program on Space Science, the Chinese Academy of Science (Grant No. XDA15020302).

\section{REFERENCES}

Blaauw R. C., 2017, Planet. Space Sci., 143, 83

Boice D. C., 2017, J. Appl. Math. Phys., 5, 311

Boice D. C., Benkhoff J., 2015, Modelling the Near-Sun Object, 3200 Phaethon, 46th LPSC, Contrib. No. 1832
Borovička J., Koten P., Spurny P., Čapek D., Shrbený L., Štork R., 2010, Material properties of transition objects 3200 Phaethon and 2003 EH1, Icy Bodies of the Solar System. Proc. IAU Symp. 263, p. 218

Bowell E., Hapke B., Domingue D., Lumme K., Peltoniemi J., Harris A. W., 1989, Asteroids II. Univ. Arizona Press, Tucson, AZ, p. 524

Carry B. et al., 2010, Icarus, 205, 460

Chamberlin A. B., McFadden L.-A., Schulz R., Schleicher D. G., Bus S. J., 1996, Icarus, 119, 173

de León J. et al., 2010, A\&A, 513, A26

Epstein P. S., 1924, Phys. Rev., 23, 710

Green S. F., 1983, IAU Circ. No. 3878.

Gundlach B., Blum J., 2013, Icarus, 223, 479

Gustafson B. A. S., 1989, A\&A, 225, 533

Hanuš J. et al., 2016, A\&A, 592, A34

Hsieh H., Jewitt D., 2006, Science, 312, 561

Hui M. T., Li J., 2017, ApJ, 153, 23

Ito T. et al., 2018, Nat. Commun., 9, 2486

Jenniskens P., 2008, Earth Moon Planets, 102, 505

Jewitt D., 2013, AJ, 145, 133

Jewitt D., Li J., Agarwal J., 2013, ApJ, 771, L35

Jewitt D., Hsieh H., Agarwal J., 2015, in Michel P., DeMeo F. E., Bottke W. F., eds, Asteroids IV. Univ. Arizona Press, Tucson, AZ, p. 221

Krokosky E. M., Husak A., 1968, J. Geophys. Res., 71, 2237

Li J., Jewitt D., 2013, AJ, 145, 154

Licandro J. et al., 2007, A\&A, 461, 751

Molaro J. L., Byrne S., Langer S. A., 2015, J. Geophys. Res., 120, 255

Podolak M., Flandes A., Corte V. D., Krüger H., 2016, Planet. Space Sci., 133,85

Takir D. et al., 2018, 3- $\mu \mathrm{m}$ Spectroscopy of Asteroid (3200) Phaethon: Implications for B-Asteroids, 49th LPSC, Contrib. No. 2083.

Rivkin A. S., Howell E. S., Vilas F., Lebofsky L. A., 2002, in Bottke W. F., Jr, Cellino A., Paolicchi P., Binzel R. P., eds, Asteroids III. Univ. Arizona Press, Tucson, AZ, p. 235

Sasselov D. D., Lecar M., 2000, ApJ, 528, 995

Schorghofer N., 2008, ApJ, 682, 697

Todorović N., 2018, MNRAS, 475, 601

Yanagisawa M., Ikegami H., Ishida M., Karasaki H., Takahashi J., Kinoshita K., Ohnishi K., 2008, Meteorit. Planet. Sci., 43, 5169

This paper has been typeset from a $\mathrm{T}_{\mathrm{E}} \mathrm{X} / \mathrm{LT}_{\mathrm{E}} \mathrm{X}$ file prepared by the author. 